\title{
Strongly confined HgTe 2D nanoplatelets as narrow near infrared emitters
}

Eva Izquierdo ${ }^{1}$, Adrien Robin ${ }^{1}$, Sean Keuleyan, ${ }^{2}$ Nicolas Lequeux ${ }^{1}$, Emmanuel Lhuillier ${ }^{3}$, Sandrine Ithurria $^{1}$ *

${ }^{1}$ Laboratoire de Physique et d'Étude des Matériaux, PSL Research University, CNRS UMR 8213, ESPCI ParisTech, 10 rue Vauquelin, 75005 Paris, France.

${ }^{2}$ Voxtel, Inc., University of Oregon, CAMCOR, 1241 University of Oregon, Eugene, OR 97403, USA

${ }^{3}$ Sorbonne Universités, UPMC Univ Paris 06, CNRS-UMR 7588, Institut des Nanosciences de Paris, F-75005 Paris, France.

*To whom correspondence should be sent: sandrine.ithurria@espci.fr

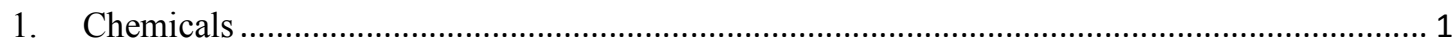

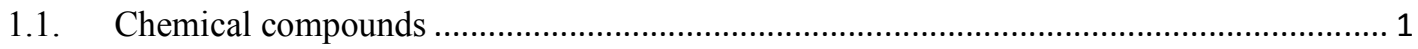

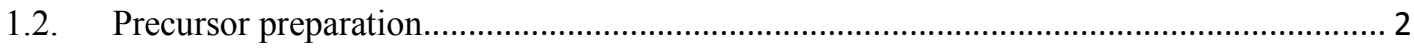

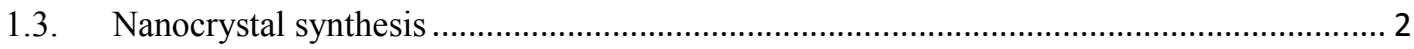

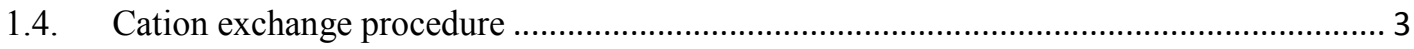

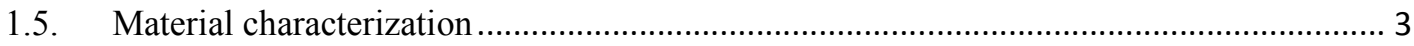

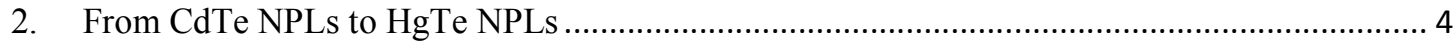

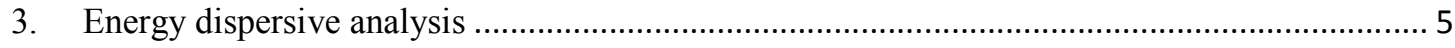

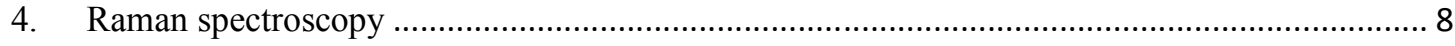

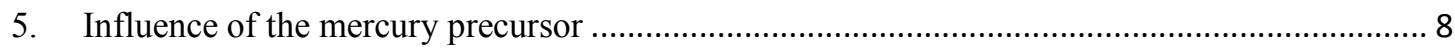

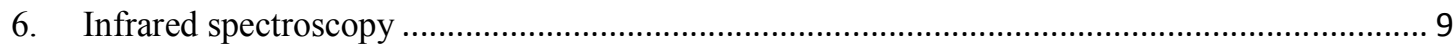

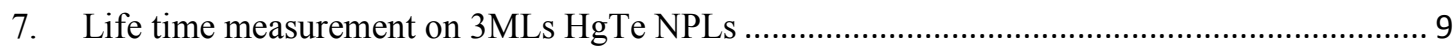

8. Electrolytic field effect transistor ....................................................................................... 10

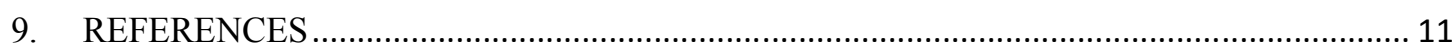

\section{Chemicals}

\subsection{Chemical compounds}

Te powder (Alfa-Aesar, $99.999 \%$ ), Se powder (Sigma-Aldrich), cadmium oxide (CdO) (Aldrich $99.99 \%$ ), $\mathrm{LiClO}_{4}$ (Aldrich, $98 \%$ ), $\mathrm{Hg}(\text { Acetate })_{2}$ ( $\mathrm{Hg}(\mathrm{Ac})_{2}$ Aldrich, > $99.0 \%$ ), Oleylamine (Acros $80-$ $90 \%$ ), Octylamine (Aldrich, 99\%), trioctylphosphine (TOP) (Lancaster, $90 \%$ ), propionic acid (Aldrich, $99 \%$ ), dodecanthiol (sigma-Aldrich, $98 \%$ ), polyethylene glycol $\mathrm{M}_{\mathrm{W}}=6 \times 10^{3} \mathrm{~g} \cdot \mathrm{mol}^{-1}$ (PEG 6k) (Fluka), oleic acid (Aldrich $90 \%$ ), indocyanine green (ICG) (Sigma-Aldrich, $80 \%$ ), Trioctylamine (Aldrich), Octadecene (ODE) (Aldrich, $90 \%$ ), toluene (VWR, 99.8 \%), ethanol (Carlo Erba, $99.5 \%$ ), n-Hexane (VWR, $98 \%$ ), acetone (Sigma-Aldrich, > $99.5 \%$ ), Dimethylsulfoxide (DMSO) (Sigma-Aldrich, > $99.5 \%$ ). 


\subsection{Precursor preparation}

- Cadmium propionate

Cadmium propionate is obtained by mixing $1.036 \mathrm{~g}$ of $\mathrm{CdO}$ powder with $10 \mathrm{~mL}$ of propionic acid under Ar for $1 \mathrm{~h}$. The flask is then open and heated at $140^{\circ} \mathrm{C}$ until the volume gets reduced by a factor two. The white solution is precipitated with acetone. The solution is centrifuged and the supernatant discarded. The solid is dried under vacuum at room temperature for $24 \mathrm{~h}$.

- $\quad$ TOPTe (1M)

In a three neck flask, $2.54 \mathrm{~g}$ of Te powder are mixed with $20 \mathrm{~mL}$ of trioctylphosphine and degassed under vacuum at room temperature until the pressure gets reduced below $1 \mathrm{mbar}$. Then the flask is heated under $\mathrm{Ar}$, at $275^{\circ} \mathrm{C}$ until the black Te powder gets fully dissolved. The orange solution is then cooled and turns yellow. Once the temperature is below $40^{\circ} \mathrm{C}$, the solution is further degassed under vacuum for $10 \mathrm{~min}$ and finally stored in an air free glove box.

- TOPSe (1M)

In the glove box, $1.44 \mathrm{~g}$ of Se powder are mixed with $20 \mathrm{~mL}$ of trioctylphosphine and let stir until complete dissolution of Se in TOP.

\subsection{Nanocrystal synthesis}

- Synthesis of 3MLs CdTe nanoplatelets $\left(1^{\text {st }}\right.$ excitonic peak at $\left.500 \mathrm{~nm}\right)$

The procedure follows the procedure proposed by Pedetti et al ${ }^{1}$. Briefly, $260 \mathrm{mg}$ of Cd(propionate) 2 , $160 \mu \mathrm{L}$ of oleic acid and $20 \mathrm{~mL}$ of octadecene are degassed under vacuum at $90^{\circ} \mathrm{C}$ for 1 hour. Then under Argon flow, $200 \mu \mathrm{L}$ of TOPTe at $1 \mathrm{M}$ mixed with $400 \mu \mathrm{L}$ of octadecene are swiftly injected at $215^{\circ} \mathrm{C}$. After 30 minutes of reaction, the mixture is cooled down and $1 \mathrm{~mL}$ of oleic acid is injected. The resulting nanoplatelets are precipitated twice with an excess of ethanol and are redispersed in 10 $\mathrm{mL}$ of hexane.

The obtained nanocrystals have a 2D shape with typical dimension of $1.1 \mathrm{~nm}$ x $70 \mathrm{~nm}$ x $140 \mathrm{~nm}$ (see Figure S2 b and c). Their optical absorbance is composed of two main features, see Figure S2 a. The one associated with the lowest energy appear at $500 \mathrm{~nm}$. The zinc blende crystalline nature of the NPL is shown by their XRD pattern given in Figure S3.

The thickness of the nanoplatelets follows the [001] direction and is composed of 7 plans of atoms, an alternation of 4 plans of cadmium and 3 plans of tellurium. If we suppose that the $0.2 \mathrm{mmol}$ of telluride has reacted, in that case $0.26 \mathrm{mmol}$ of cadmium has also reacted. And the concentration of cadmium in the solution of CdTe NPLs is $26 \mathrm{mM}$.

\section{- Synthesis of 3 MLs CdSe nanoplatelets $\left(1^{\text {st }}\right.$ excitonic peak at $\left.462 \mathrm{~nm}\right)$}

In a three neck flask $240 \mathrm{mg}$ of $\mathrm{Cd}(\mathrm{Ac})_{2}, 2 \mathrm{H}_{2} \mathrm{O}, 150 \mu \mathrm{L}$ of oleic acid and $15 \mathrm{ml}$ of octadecene are degassed under vacuum at $80^{\circ} \mathrm{C}$ during 1 hour. Then under argon flow, the mixture is heated up to $195^{\circ} \mathrm{C}$ and $150 \mu \mathrm{L}$ of TOPSe at $1 \mathrm{M}$ are swiftly injected. After 30 minutes the mixture is cooled down and the 3 MLs are precipitated with ethanol and suspended in $10 \mathrm{~mL}$ of hexane ${ }^{2}$.

\section{- Synthesis of 2 MLs CdTe nanoplatelets ( $\left(1^{\text {st }}\right.$ excitonic peak at $\left.428 \mathrm{~nm}\right)$}

The follow procedure is also proposed by Pedetti et al. ${ }^{1}$. In a three neck flask, $130 \mathrm{mg}$ of $\mathrm{Cd}$ (propioniate) $)_{2}$ and $80 \mu \mathrm{L}$ of oleic acid are added in $10 \mathrm{~mL}$ of octadecene. The mixture is degassed at $90^{\circ} \mathrm{C}$ for 1 hour. Then under Argon flow, $100 \mu \mathrm{L}$ of TOPTe at $1 \mathrm{M}$ mixed with $500 \mu \mathrm{L}$ of octadecene are swiftly injected at $180^{\circ} \mathrm{C}$. After 20 minutes of reaction, the mixture is cooled down and $1 \mathrm{~mL}$ of oleic acid is injected. The obtained nanoplatelets are precipitated twice with an excess of ethanol and are redispersed in $10 \mathrm{~mL}$ of hexane. 


\subsection{Cation exchange procedure}

\section{- Cation exchange with $\mathrm{HgAc}_{2}$ in octylamine}

In order to show the interest of "bulky" precursors, we have performed cation exchange with $\mathrm{Hg}($ Acetate) 2 in octylamine (C8). On figure S1 we observe a redshift of the first exciton peak then a decrease of its intensity. This is due to the dissolution of the obtained HgTe NPLs.

In $2 \mathrm{~mL}$ of hexane, $20 \mu \mathrm{L}$ of CdTe nanoplatelets are introduced. At room temperature, $100 \mu \mathrm{L}$ of a freshly prepared solution at $10 \mathrm{mM}$ of $\mathrm{HgAc}_{2}$ in octylamine are introduced. Kinetic monitoring is shown on Figure S1.

\section{- Cation exchange with $\mathrm{Hg}(\mathrm{Ac})_{2}$ in oleylamine}

A solution of variable concentrations of $\mathrm{HgAc}_{2}$ in oleylamine are freshly prepared. In a flask, $2 \mathrm{~mL}$ of hexane and $20 \mu \mathrm{L}$ of CdTe nanoplatelets are introduced. Then $100 \mu \mathrm{L}$ of a solution at $10 \mathrm{mM}$ of $\mathrm{HgAc}_{2}$ in oleylamine is introduced at room temperature (the ratio $\mathrm{Cd}: \mathrm{Hg}$ is $1: 2$ ). Absorption spectra are acquired to follow the cation exchange. Once the first exciton has reached $880 \mathrm{~nm}$, the nanoplatelets are precipitated and resuspended in hexane. Regarding the large laterals sizes, the nanoplatelets precipitate without addition of a polar solvent. If a large excess of $\mathrm{HgAc}_{2}$ in oleylamine is used, the HgTe nanoplatelets get dissolved by oleylamine. Emission spectra of the nanoplatelets during the cation exchange are presented in figure S11.

\section{- Cation exchange with $\mathrm{Hg}(\mathrm{Ac})_{2}$ in trioctylamine}

In a flask, $2 \mathrm{~mL}$ of hexane and $20 \mu \mathrm{L}$ of CdTe nanoplatelets are introduced. At room temperature, 40 $\mu \mathrm{L}$ of a freshly prepared solution at $25 \mathrm{mM}$ of $\mathrm{HgAc}_{2}$ in trioctylamine are introduced (the ratio $\mathrm{Cd}: \mathrm{Hg}$ is 1:2). Absorption is used to follow the cation exchange. Once the first excitonic peak has reach 822 $\mathrm{nm}, 20 \mu \mathrm{L}$ of oleic acid are injected to stabilize the HgTe NPLs. The excess of precursors are removed by centrifugation and washed with ethanol. The nanoplatelets are then resuspended in hexane for further experiments. Kinetic monitoring is shown on Figure S11.

\section{- Cation exchange with $\mathrm{HgCl}_{2}$ in oleylamine}

In $2 \mathrm{~mL}$ of hexane, $20 \mu \mathrm{L}$ of CdTe nanoplatelets are introduced. At room temperature, $100 \mu \mathrm{L}$ of a freshly prepared solution at $10 \mathrm{mM}$ of $\mathrm{HgCl}_{2}$ in oleylamine are introduced. Kinetic monitoring is shown on Figure S10.

\subsection{Material characterization}

UV-visible spectrum have been acquired from a Shimadzu UV-1800 spectrometer. Photoluminescence spectra are obtained from an Edinburgh Instrument spectrometer.

For Transmission electron image, the nanoparticles are dispersed in hexane and dropcast on a TEM grid. The grids are then degassed overnight under secondary vacuum. Images are acquired on a JEOL 2010 microscope.

$\mathrm{X}$ ray diffraction is conducted by dropcasting a solution of NPLs on a Si wafer. The diffractometer is a Philips X'Pert based on the emission of the $\mathrm{Cu} \mathrm{K}_{\alpha}$ line operated at $40 \mathrm{kV}$ and $40 \mathrm{~mA}$.

The photoluminescence quantum yield of HgTe NPLs has been determined in comparison to ICG reference dye in DMSO using the following expression:

$$
Q Y_{N P L S}=Q Y_{I C G} \frac{I_{N P L s}}{I_{I C G}} \frac{O D_{I C G}}{O D_{N P L s}} \frac{n_{N P L s}^{2}}{n_{I C G}^{2}}
$$


The fluorescence decay has been fitted with three exponentials as it has already been done for cadmium chalcogenides NPLs. Despite their monodispersity, the nanoplatelets exhibit multiexponentials fluorescence decay which may come from several emitting states in a single NPL ${ }^{3}$. The average life time of 50ns has been deduced from the three lifetimes regulated by the percentage of photons emitting at this life time.

\section{From CdTe NPLs to HgTe NPLs}

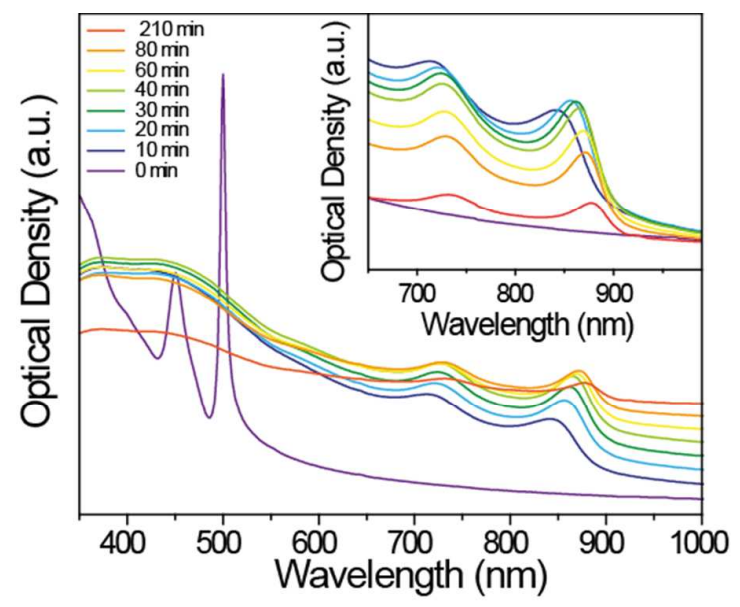

Figure S1: Absorption kinetics of cation exchange on CdTe nanoplatelets with a solution of $\mathrm{HgAc}_{2}$ in octylamine. After 210 minutes of reaction the intensity of the absorption have decreased due to the dissolution of the HgTe NPLs.

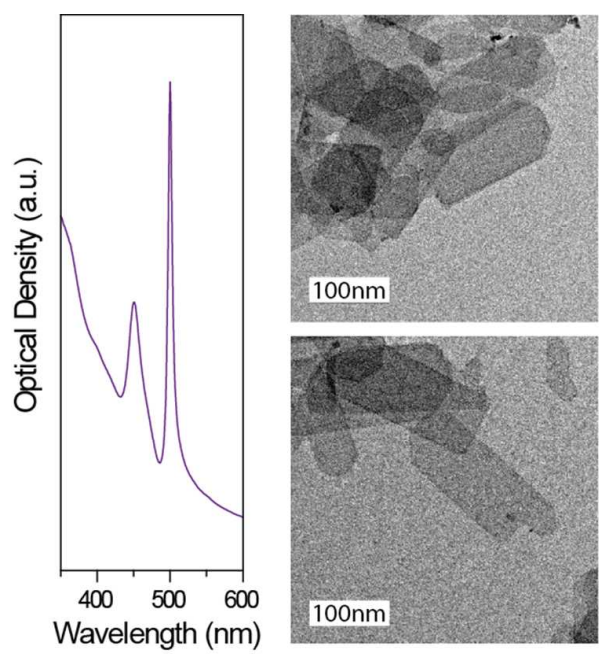

Figure S2: (a.) Absorbance spectrum of 3MLs CdTe NPLs with a first excitonic peak at $500 \mathrm{~nm}$. (b.) and (c.) transmission electron microscopy images of $3 M L s$ CdTe NPLs 

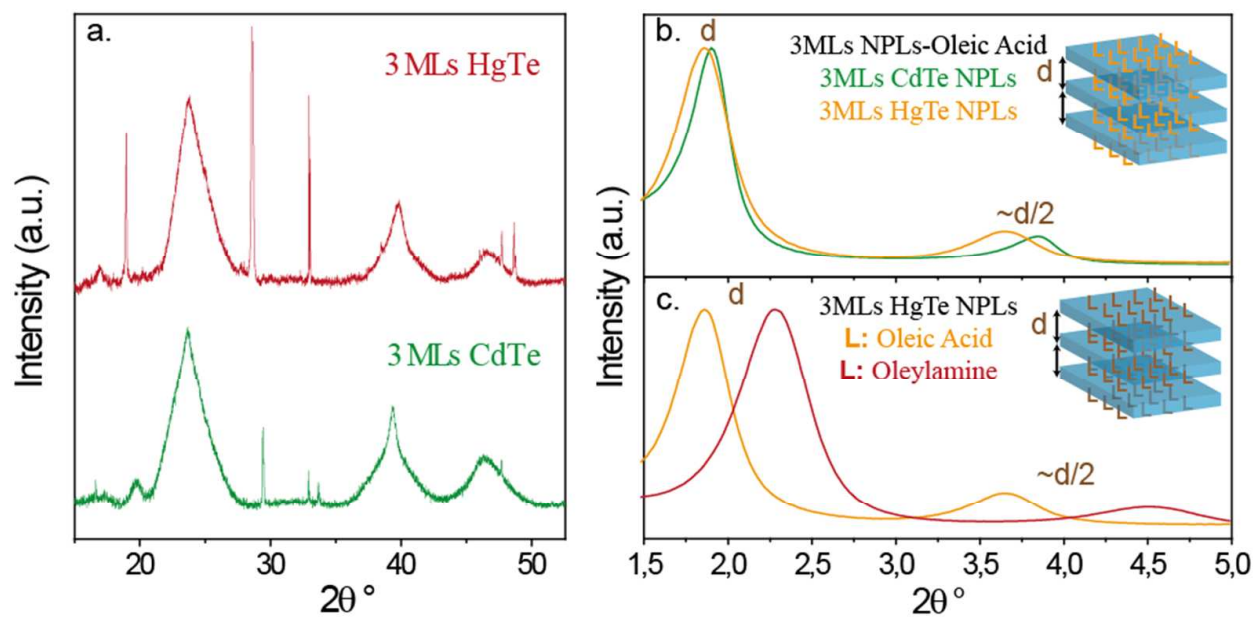

Figure S3: (a.) XRD pattern of 3MLs CdTe and HgTe NPLs. The sharp thin peaks are due to the substrate (b) Small angle diffraction of $3 M L s$ oleic acid capped CdTe (green) and HgTe (orange)

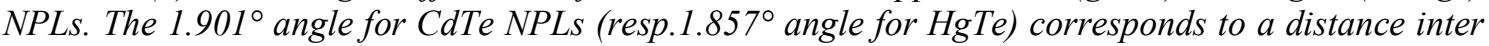
plane equal to $4.64 \mathrm{~nm}$ (resp. $4.75 \mathrm{~nm}$ ). This distance represents the thickness of the nanoplatelets $(1.1 \mathrm{~nm})$ plus the interpenetrated ligands (3.6nm) which are oleic acids.(c) Small angle diffraction of $3 M L s$ oleic acid (orange) and oleylamine (red) capped and HgTe NPLs. The $1.857^{\circ}$ angle for oleic acid capped 3MLs HgTe NPLs (resp.2.282 angle for oleylamine capped 3MLs HgTe NPLs) corresponds to a distance inter plane equal to $4.75 \mathrm{~nm}$ (resp. $3.87 \mathrm{~nm}$ ).
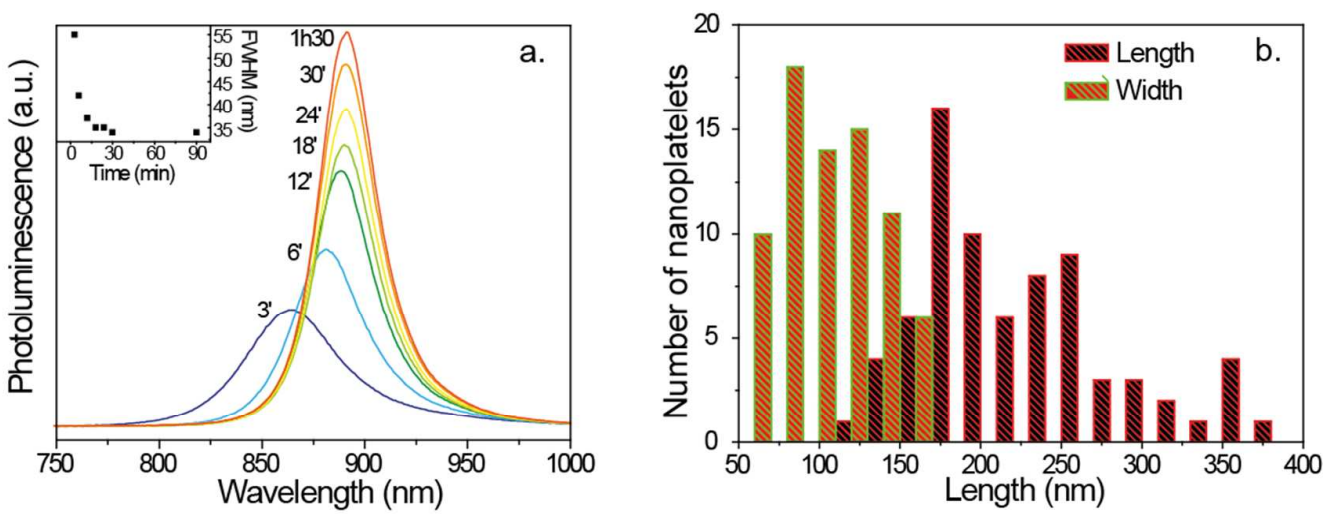

Figure S4: (a.) Emission spectra of $\mathrm{Hg}(\mathrm{Cd}) \mathrm{Te}$ to $\mathrm{HgTe}$ cation exchange with $\mathrm{Hg}(\text { Acetate })_{2}$ in oleylamine as a precursor of mercury. For clarity, the emission spectrum of the initial 3MLs CdTe NPLs is not represented.(b.) Histogram of 3MLs HgTe nanoplatelets showing averages length of 200 $\mathrm{nm}$ and width of $100 \mathrm{~nm}$.

\section{Energy dispersive analysis}

Energy Dispersive X ray spectroscopy (EDS) is performed using a Oxford EDS spectrometer in a FEI Magellan scanning electron microscope. The EDS spectrum of the CdTe and HgTe NPL thin are respectively given in Figure S5 and Figure S6. The quantitative analysis of the spectrum (see Table S1) pledge for a full cation exchange with no cadmium left or at least below the EDS resolution $(\approx 2$ $\%$ ). The EDX spectrum of CdSe and HgSe NPLs are also acquired (see respectively Figure S7 and S8 and Table S2). 


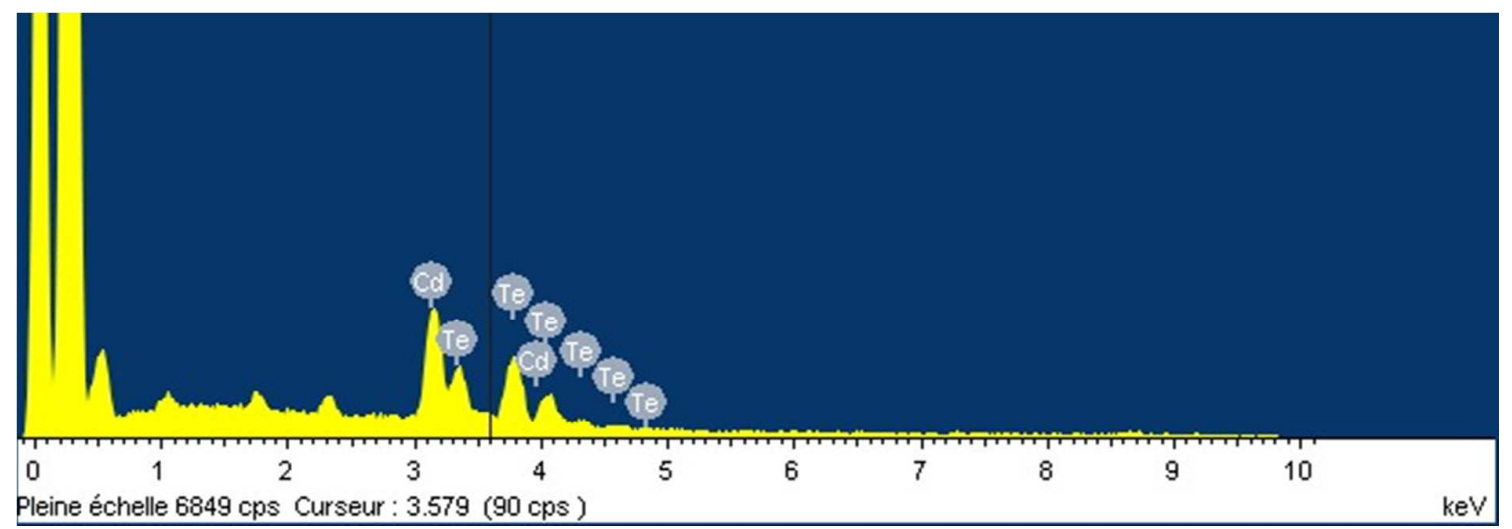

Figure S5: EDS spectrum of a CdTe NPLs film.

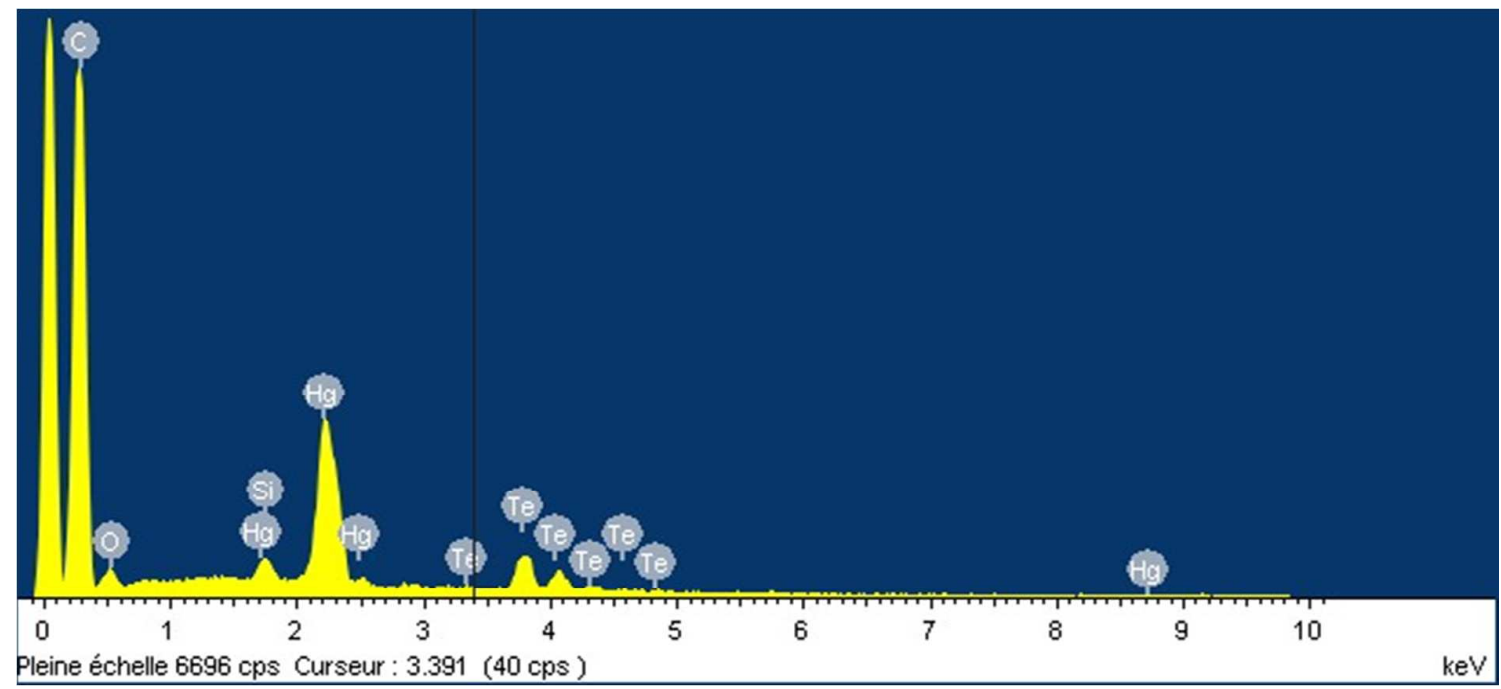

Figure S6: EDS spectrum of a HgTe NPLs film (HgTe-TOAm capped with Oleic acid).

Table S1 : Quantitative composition analysis of CdTe and $\mathrm{HgTe}$ thin film made from EDS

\begin{tabular}{lll}
\hline \hline Sample & $\begin{array}{l}\text { metal ratio on atoms content } \\
\mathbf{( \% )}\end{array}$ & $\begin{array}{l}\text { Te ratio on atoms content } \\
\mathbf{( \% )}\end{array}$ \\
\hline CdTe & $53 \pm 2$ & $47 \pm 2$ \\
\hline HgTe - Oam exchange & $59 \pm 2$ & $41 \pm 2$ \\
\hline HgTe - TOAm exchange & $59 \pm 2$ & $41 \pm 2$ \\
\hline \hline
\end{tabular}




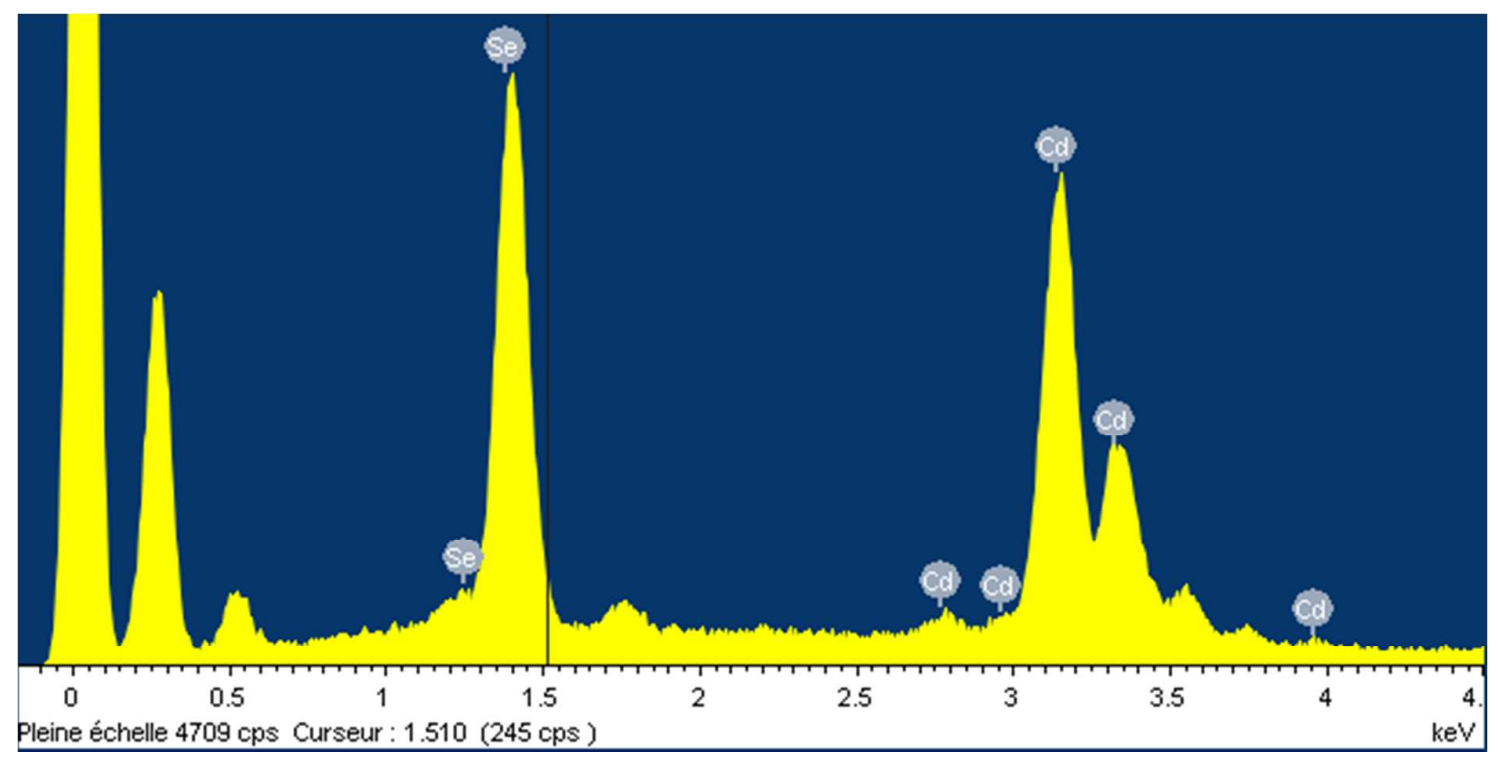

Figure S7: EDS spectrum of a CdSe (3 MLs) NPLs film.

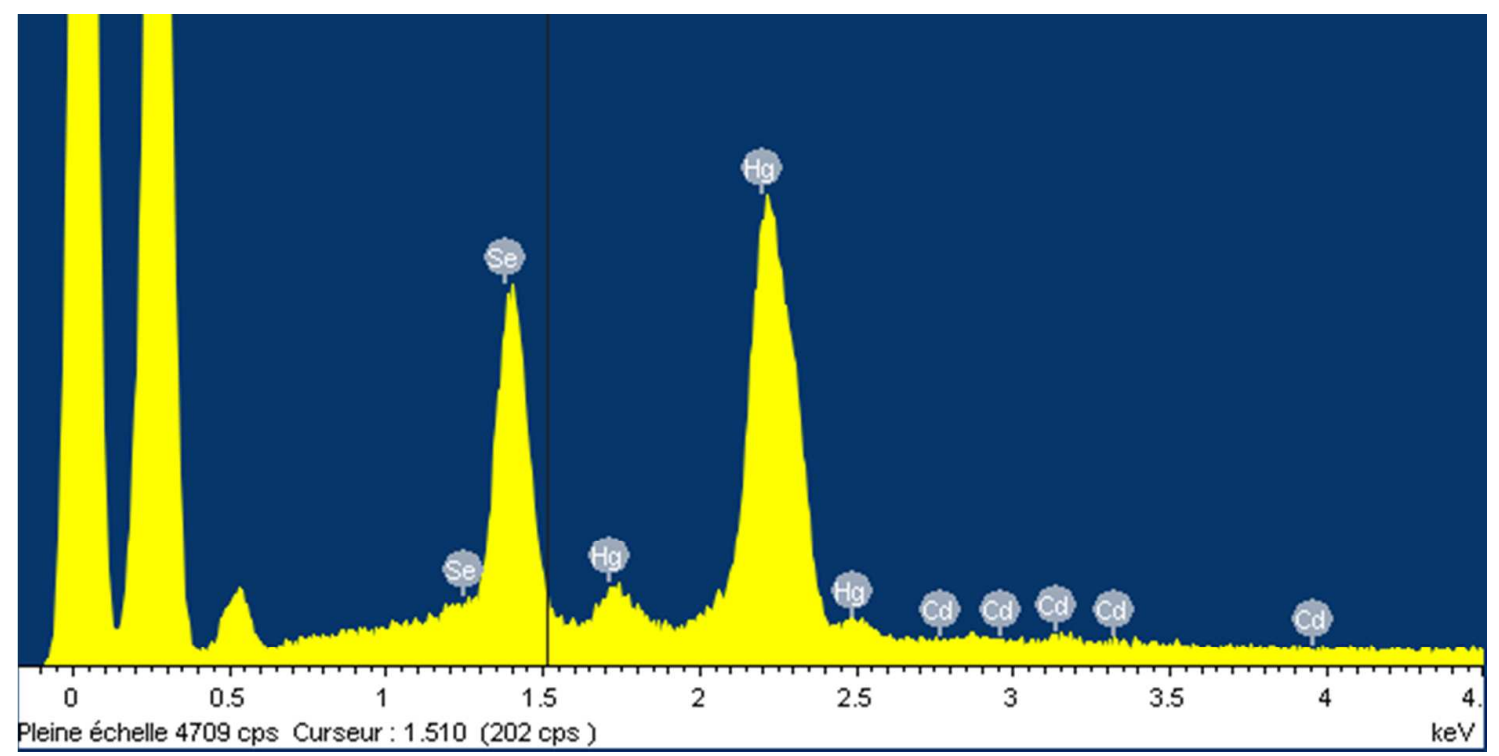

Figure S8: EDS spectrum of a HgSe NPL film.

Table S2 : Quantitative composition analysis of CdSe and HgSe thin film made from EDS

\begin{tabular}{lll}
\hline \hline Sample & $\begin{array}{l}\text { metal ratio on atoms content } \\
(\mathbf{\%})\end{array}$ & Se ratio on atoms content (\%) \\
\hline $\mathrm{CdSe}$ & $53 \pm 2$ & $47 \pm 2$ \\
\hline $\mathrm{HgSe}-$ Oam exchange & $55 \pm 2$ & $45 \pm 2$ \\
\hline \hline
\end{tabular}




\section{Raman spectroscopy}

For Raman spectroscopy, we dropcast some NPLs solution on a Si substrate. Raman spectra are acquired from a nanoparticle film deposited on a Si substrate using a confocal commercial Renishaw micro-Raman microscope with a $100 \times$ objective, a Si detector, and a $532 \mathrm{~nm}$ laser excitation.

Figure S9 presents the low energy Raman spectra for the CdTe NPLs film before and after cation exchange with mercury. The main feature relative to the LO phonon of CdTe at $166 \mathrm{~cm}^{-1}$ is fully removed after cation exchange.

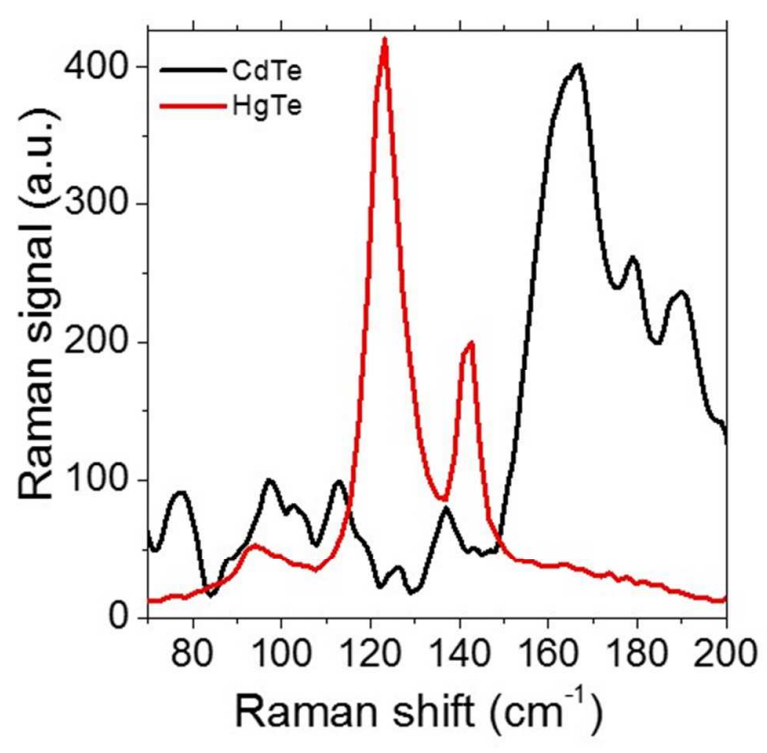

Figure S9: Low energy background subtracted Raman spectra of CdTe and HgTe NPL film. Illumination is based on a $532 \mathrm{~nm}$ laser diode.

\section{Influence of the mercury precursor}

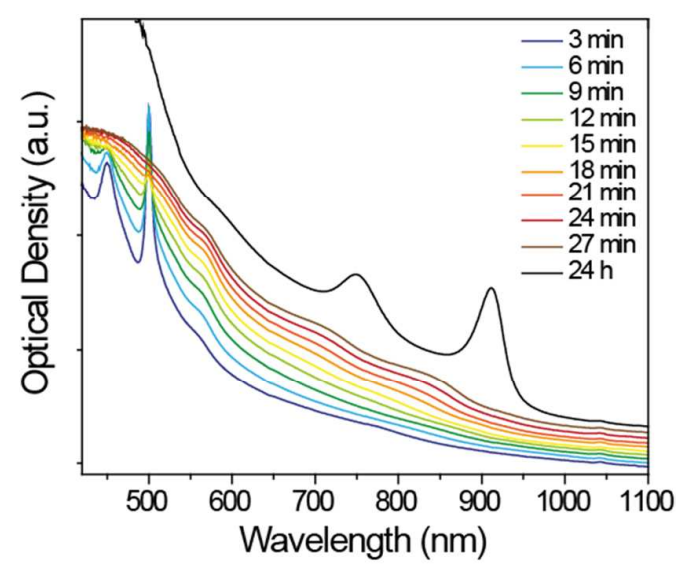

Figure S10: Absorption kinetics of cation exchange on CdTe nanoplatelets with a solution of $\mathrm{HgCl}_{2}$ in oleylamine. 


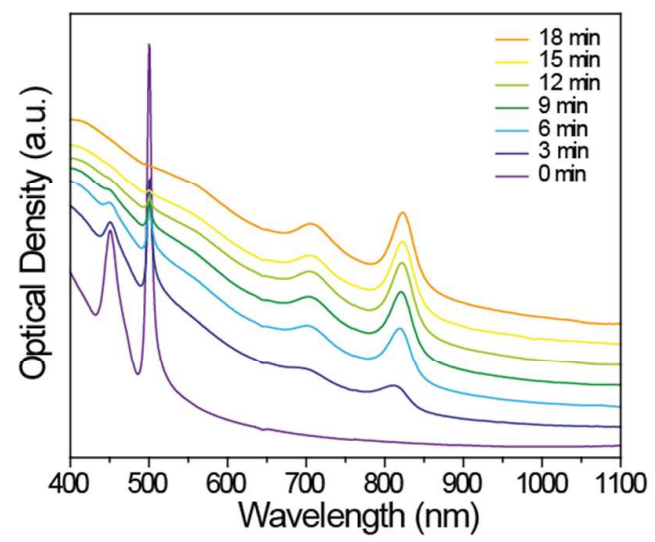

Figure S11: Absorption spectra of CdTe to $\mathrm{HgTe}$ cation exchange with $\mathrm{Hg}(\mathrm{Ac})_{2}$ in trioctylamine as a precursor of mercury.

\section{Infrared spectroscopy}

The infrared spectra have been acquired on a Bruker vertex 70 equipped with an ATR. On the following figure S12, we show the absorption infrared spectra for HgTe 3MLs nanoplatelets capped with oleic acid, oleylamine or dodecanethiol. The change from oleic acid to oleylamine ligand is accompanied by the disappearance of the $\mathrm{C}=\mathrm{O}, \mathrm{CO}_{2}$ stretches and the appearance of the $\mathrm{NH}$ stretch. While changing from oleylamine to dodecanethiol, the $\mathrm{NH}$ stretch disappears.

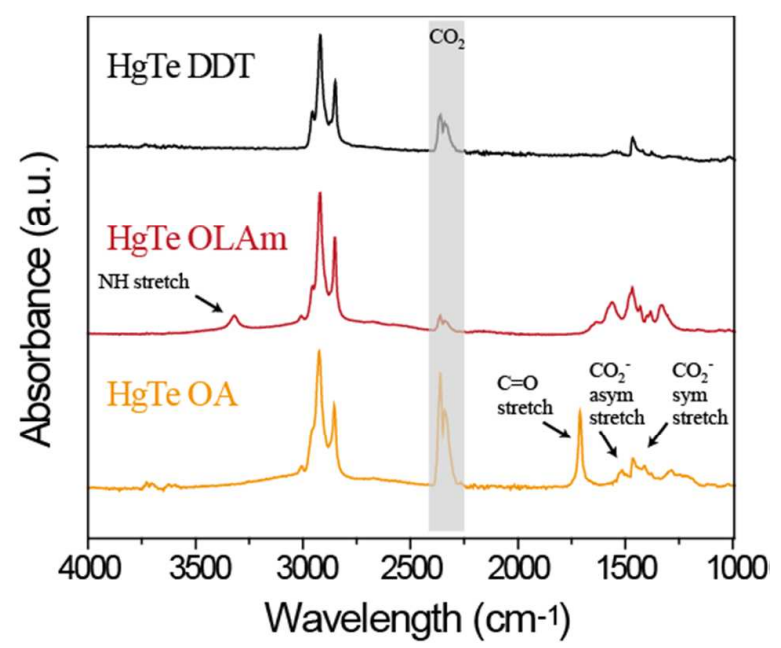

Figure S12: IR spectra of HgTe capped with oleic acid (black), oleylamine (red) and dodecanethiol (green).

\section{Life time measurement on 3MLs HgTe NPLs}

The life time measurement has been acquired with an Edinburgh Instrument spectrometer. The fluorescence decay has been fitted with three exponentials as it has already been done for cadmium chalcogenides NPLs (see Figure S13). Despite their monodispersity, the nanoplatelets exhibit multiexponentials fluorescence decay which may come from several emitting states in a single $\mathrm{NPL}^{3}$. 
The average life time of $50 \mathrm{~ns}$ has been deduced from the three lifetimes regulated by the percentage of photons emitting at this life time.

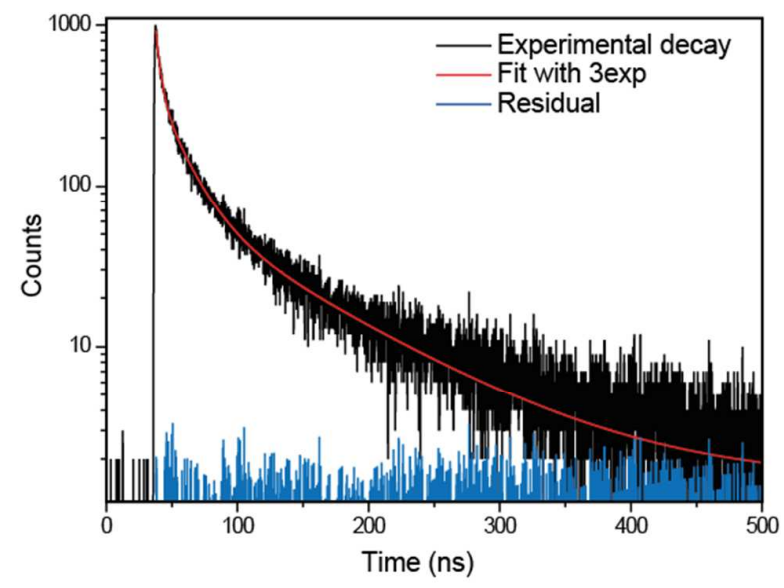

$$
f(t)=B_{1} e^{-t / \tau 1}+B_{2} e^{-t / \tau 2}+B_{3} e^{-t / \tau 3}
$$

\begin{tabular}{cccc}
\hline $\boldsymbol{i}$ & $\mathbf{B}$ & $\boldsymbol{\tau}$ (ns) & Amplitude $\%$ \\
\hline $\mathbf{1}$ & 530 & 3 & 11 \\
$\mathbf{2}$ & 322 & 19 & 43 \\
$\mathbf{3}$ & 75 & 89 & 46 \\
\hline
\end{tabular}

$$
\tau_{\text {avg }}=\langle\tau\rangle=\sum_{i=1,2,3} \tau_{i} f_{i} \approx 50 n s
$$

Figure S13: PL decay curve with three exponential fit of 3MLs HgTe NPLs capped with oleic acid. The decay constants are: $3 \mathrm{~ns}$ (11\% of amplitude), $19 \mathrm{~ns}$ (43\% of amplitude) and $89 \mathrm{~ns}$ (46\% amplitude). The average lifetime is 50ns.

\section{Electrolytic field effect transistor}

We first prepare in the glove box electrolyte my melting $\mathrm{LiClO}_{4}(0.5 \mathrm{~g})$ with $2.3 \mathrm{~g}$ of polyethylene glycol $\left(\mathrm{M}_{\mathrm{W}}=6 \mathrm{~kg} \cdot \mathrm{mol}^{-1}\right)$ at $170^{\circ} \mathrm{C}$ on a hot plate for $2 \mathrm{~h}$. Meanwhile, interdigitated electrodes are prepared in the cleanroom using standard lithography method. Typically, on a $\mathrm{Si} / \mathrm{SiO}_{2}$ substrate, we prepare 25 pairs of interdigitated electrodes with a $20 \mu \mathrm{m}$ spacing and $2 \mathrm{~mm}$ length. The electrodes are made of $3 \mathrm{~nm} \mathrm{Cr}$ and $40 \mathrm{~nm}$ gold.

The ligands of the NPL are then exchanged toward $\mathrm{S}^{2-}$ capping using a phase transfer procedure ${ }^{4}$. The nanoparticle dispersed in n-methyl formamide and then dropcast on the electrodes on a hot plate at $100^{\circ} \mathrm{C}$ in the glove box. Once the film is dried, the warm electrolyte is brushed on the film. Finally, a metallic grid is deposited and used as top gate. Electrical measurements are conducted using a homemade probe station connected to two Keithley 2400 sourcemeters. 

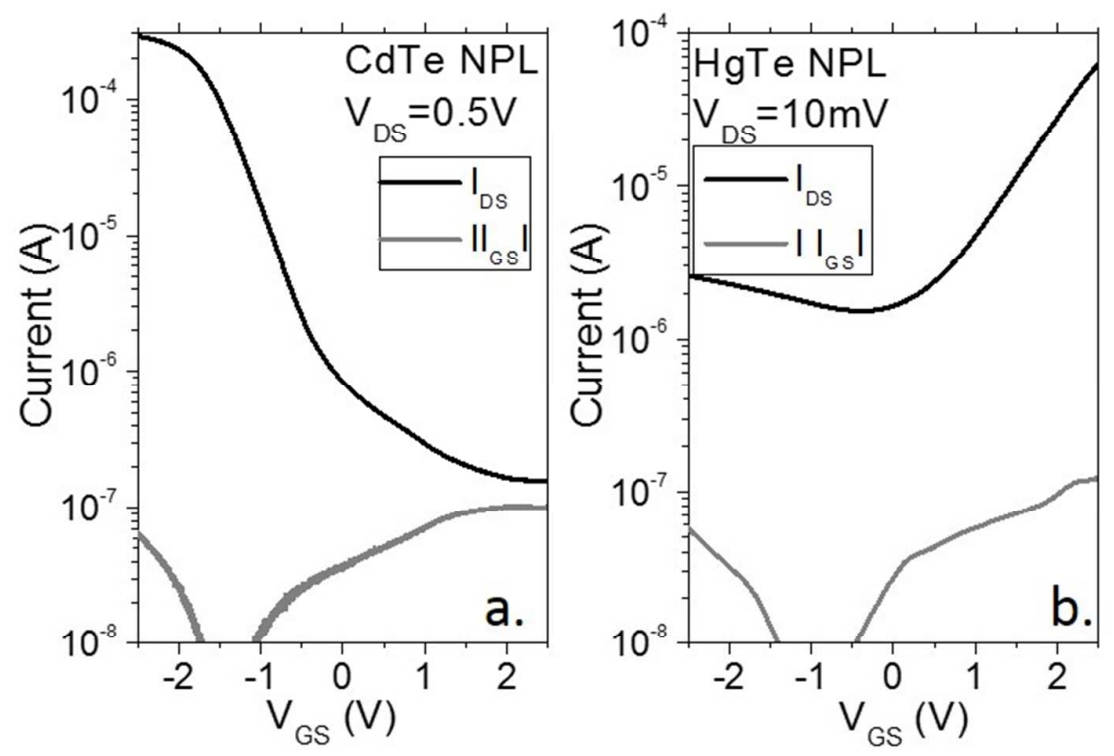

Figure S14: Drain and gate current in logarithmic scale for CdTe (a) and HgTe thin film gated suing ion gel electrolyte. The applied drain source bias is $0.5 \mathrm{~V}$ for CdTe and $10 \mathrm{mV} \mathrm{for} \mathrm{HgTe.} \mathrm{The} \mathrm{gate} \mathrm{step}$ bias is $1 \mathrm{mV}$. The sweep rate is $2 \mathrm{mV} \cdot \mathrm{s}^{-1}$.

\section{REFERENCES}

(1) Pedetti, S.; Nadal, B.; Lhuillier, E.; Mahler, B.; Bouet, C.; Abécassis, B.; Xu, X.; Dubertret, B. Chem. Mater. 2013, 25 (12), 2455.

(2) Ithurria, S.; Bousquet, G.; Dubertret, B. J. Am. Chem. Soc. 2011, 133 (9), 3070.

(3) Tessier, M. D.; Javaux, C.; Maksimovic, I.; Loriette, V.; Dubertret, B. ACS Nano 2012, 6 (8), 6751.

(4) Nag, A.; Kovalenko, M. V; Lee, J.; Liu, W.; Spokoyny, B.; Talapin, D. V. J. Am. Chem. Soc. 2011, 133 (27), 10612. 